\title{
Implantação de stent autoexpansível para tratamento de estenose uretral em cão: relato de caso
}

\author{
[Implantation of self-expanding stent for treatment of urethral stenosis in dog - case report] \\ A.A. Ferreira ${ }^{1}$, N.N. Kano $^{2}$, L. Ozório ${ }^{3}$, P.F. Freitas ${ }^{3}$, R. Carareto $^{4}$, \\ J.L.C. Castro ${ }^{5}$, T.R. Froes ${ }^{4}$, P.T. Dornbusch ${ }^{4}$. \\ ${ }^{1}$ Aluna de Pós-graduação - Universidade Federal do Paraná - Curitiba, PR \\ ${ }^{2}$ Aluna de graduação - Universidade Federal do Paraná - Curitiba, PR \\ ${ }^{3}$ Residente - Universidade Federal do Paraná - Curitiba, PR \\ ${ }^{4}$ Universidade Federal do Paraná - Curitiba, PR \\ ${ }^{5}$ Pontifícia Universidade Católica do Paraná - Curitiba, PR
}

\author{
A.A. Ferreira1 \\ https://orcid.org/0000-0002-1576-9547 \\ N.N. Kano2 \\ https://orcid.org/0000-0002-7132-7073 \\ L. Ozorio3 \\ https://orcid.org/0000-0002-9324-0243
P.F. Freitas3 \\ P.F. Freitas3 3 \\ https://orcid.org/0000-0002-3575-4430 \\ R. Carareto4 \\ J.L.C. Castro5 \\ J.L.C. Castro5
https://orcid.org/0000-0001-5496- 8912 \\ https://orcid.org/ \\ https://orcid.org/0000-0002-7802-2598 \\ P.T. Dornbusch4 \\ https://orcid.org/0000-0002-0280-6548
}

\section{RESUMO}

O stent endovascular autoexpansível pode ser utilizado como forma de tratamento para estenose uretral em cães. O stent de nitinol é composto por uma liga de níquel e titânio que permite mudanças em suas estruturas sob a influência da temperatura corporal e tem excelente biocompatibilidade na uretra canina. Este estudo relata o caso de um cão, sem raça definida, 14 anos de idade, com histórico de disúria há duas semanas, diagnosticado com grande quantidade de cálculos e microcálculos na bexiga, no colo vesical e na uretra prostática, também cistite e hiperplasia prostática. Depois de tratamento para cistite, orquiectomia e cistotomia para retirada dos cálculos, porém sem melhora clínica após 30 dias de acompanhamento, com o paciente apresentando disúria, foi realizada uretrocistografia retrógrada, que evidenciou estenose da uretra prostática. Esse paciente foi encaminhado para procedimento de implantação de um stent de nitinol na uretra prostática. Imediatamente após o procedimento e até o presente momento, o paciente apresenta micção espontânea, sem qualquer sinal de disúria, sendo este o primeiro relato no Brasil do uso dessa técnica.

Palavras-chave: stent endovascular autoexpansível, uretra prostática, disúria

\begin{abstract}
The endovascular stent is used for treatment of urethral stricture in dogs. The nitinol stent is composed by nickel and titanium alloy that allows changes in its structures under the influence of body temperature and has excellent biocompatibility in the canine urethra. This study aims to report a case of a 14 - year old male dog without defined breed with dysuria for two weeks. Ultrasonographic evaluation revealed microcalculi and calculi in bladder, prostatic urethral obstruction and prostatic hyperplasia. Cystotomy was performed to remove uroliths, but after 30 days of hospitalization there was no clinical improvement. A double-contrast retrograde urethrocystography was performed and showed persistence of prostatic urethral obstruction. The pacient was submitted to a new surgery to place a self-expanding nitinol stent and presented spontaneous urination after the procedure. This is the first report of this technique in Brazil.
\end{abstract}

Keywords: endovascular self-expanding stent, prostatic urethra, dysuria

\section{INTRODUÇÃO}

O primeiro relato do uso de stent vascular para tratamento de estenose uretral em cães foi feito por Milroy et al. (1988), que o implantaram na

Recebido em 16 de novembro de 2017

Aceito em 27 de agosto de 2018

E-mail: arieleferreira@hotmail.com uretra de quatro cães e demostraram tratar-se de um tratamento viável para estenose uretral. O stent pode ser implantado de forma temporária ou permanente (Latal et al., 1994; Liatsikos et al., 2007), sendo considerado o tratamento de escolha para estenose uretral (Choi et al., 2007). Os self-expanding metallic stent (SEMS) de 
nitinol são compostos por uma liga de níquel e titânio que permite mudanças em suas estruturas sob a influência da temperatura corporal, têm sua pressão de expansão predefinida, são flexíveis o suficiente para se adaptarem à parede uretral, altamente resistentes à corrosão e têm excelente biocompatibilidade na uretra canina (Weisse et $a l ., 2006)$. O stent é formado por um único fio de nitinol o que diminui o risco de lesão uretral (Ko et al., 2002).

O stent uretral é implantado por uma técnica inicialmente descrita sob a orientação fluoroscópica, porém a radiografia digital é uma opção facilmente disponível na prática de pequenos animais e pode ser utilizada como alternativa à fluoroscopia, permitindo maior disponibilidade para esse procedimento (Choi et al., 2009; Radhakrshnan, 2017).

O diâmetro do stent deve ser $10 \%$ mais largo que a uretra adjacente à obstrução, e no comprimento é indicado que o stent ultrapasse $0,5 \mathrm{a} 1 \mathrm{~cm}$ para estar em contato com o tecido normal cranial e caudal à região estenosada (Weisse et al., 2006; Choi et al., 2009;; Brace et al., 2013; Hill et al., 2014; Radhakrishnan, 2017).

Em estudo realizado por Hill et al. (2014), os autores demonstram as complicações que podem ocorrer após a implantação do stent uretral, sendo a principal e mais comum a incontinência urinária, que é relatada em diversos estudos. Outras complicações seriam as obstruções recorrentes em caso de estenose por neoplasia ou por formação de tecido de granulação exacerbante no lúmen do stent, a migração do stent para a bexiga urinária ou a fratura do stent (Ko et al., 2002; Hill et al., 2014). Também podem estar relacionados, após a colocação do stent uretral, quadros de hematúria persistente, polaquiúria, estrangúria e disúria (Radhakrishnan, 2017). Em alguns casos, são necessárias novas intervenções, com substituição ou remoção do stent e ressecção do tecido de granulação, quando houver (Ko et al., 2002).

Quando ocorrem essas complicações, o stent deve ser rapidamente removido; caso haja necessidade de uso permanente, eles devem ser trocados. Os fatores de risco para complicações podem ser minimizados com manejo clínico adequado, como aumento da ingestão hídrica, avaliação imediata mediante as queixas clínicas e tratamento correto quando identificada infecção do trato urinário (Dyer et al., 2002).

\section{RELATO DE CASO}

Foi atendido, no Hospital Veterinário da Universidade Federal do Paraná (HV- UFPR), um cão, sem raça definida, macho, de 14 anos de idade, não castrado, que apresentava disúria há duas semanas, com tratamento prévio realizado por colega sem sucesso. Durante exame físico, observou-se dor abdominal, distensão abdominal e gotejamento urinário por repleção vesical, sem demais alterações. Exames laboratoriais demonstravam hemograma com linfopenia 511 $(/ \mu \mathrm{L})$. Os exames bioquímicos indicavam uremia com creatinina $6,6 \mathrm{mg} / \mathrm{dL}$, ureia $218,4 \mathrm{mg} / \mathrm{dL}$ e aumento de fosfatase alcalina 279,6UI/L. Urinálise demonstrava urina de coloração vermelha com aspecto hemorrágico, densidade urinária 1,012, proteinúria $(+1)$, leucocitúria (68/campo), bacteriúria $(+1)$ e incontáveis hemácias. Foi realizada urocultura e antibiograma com crescimento profuso de Streptococcus sp., tendo sido iniciada terapia para infecção baseada no antibiograma. Em ecografia abdominal, visualizava-se a bexiga com importante repleção, parede apresentando espessamento difuso, contorno irregular, preenchida por conteúdo anecoico com grande quantidade de cálculos e microcálculos, moderada quantidade de gás no lúmen vesical, colo vesical dilatado e presença de microcálculos na uretra prostática. A próstata estava aumentada de tamanho com parênquima hipoecoico heterogêneo, áreas hiperecoicas com pequenos cistos entremeados e os testículos apresentavamse com nodulações.

A resolução da obstrução uretral foi realizada com sondagem uretral e uro-hidropropulsão retrógrada, seguida de procedimento cirúrgico de cistotomia para retirada desses urólitos da vesícula urinária e orquiectomia devido às alterações prostáticas e aos nódulos testiculares. Após esse procedimento, o paciente foi acompanhado durante 30 dias e houve redução do tamanho prostático, porém mantinha disúria, sendo dependente de sondagem uretral para urinar. Devido a esse quadro, foi realizada uretrocistografia retrógrada de duplo contraste, na qual se observou imagem sugestiva de estenose de uretra prostática, tendo como 
diferenciais: uretrite inflamatória severa ou neoplasia uretral (Fig. 1).

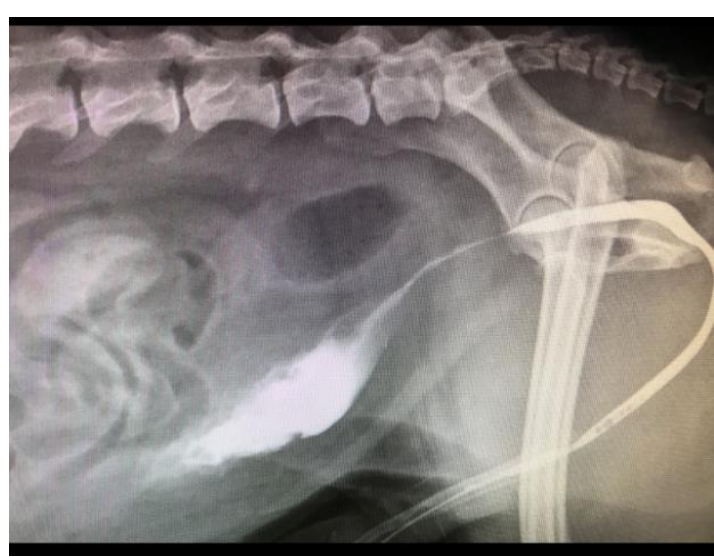

Figura 1. Uretrocistografia retrógrada de duplo contraste, com estenose da uretra prostática.

Com o quadro característico de estenose uretral, o paciente foi encaminhado para procedimento de implantação de um stent uretral. Realizou-se indução anestésica com propofol (Propovan, Cristália, Itapira, Brasil), na dose de $4 \mathrm{mg} / \mathrm{kg}$ IV, manutenção com isoflurano (Isoforine, Cristália, Itapira, Brasil) por tubo traqueal 9,0, em circuito semifechado, ventilação espontânea, fluxo de ar 2,0L/min e fiO2 100\%, analgesia com SUFLK, na dose de $5 \mathrm{~mL} / \mathrm{kg} / \mathrm{h}$, composto de citrato de sufentanila $(1 \mathrm{mcg} / \mathrm{kg} / \mathrm{h}) \quad$ (Fastfen, Cristália, Itapira, Brasil), lidocaína $2 \%$ sem vasoconstritor $(0,5 \mathrm{mg} / \mathrm{kg} / \mathrm{h})$ (Hypocaína, Hypofarma, Ribeirão das Neves, Brasil), cetamina $(0,6 \mathrm{mg} / \mathrm{kg} / \mathrm{h})$ (Quetamina injetável, Vetnil, Jacareí, Brasil), diluído em solução isotônica de cloreto de sódio 0,9\% (Fisiológico Linhamax, Eurofarma, Ribeirão Preto, Brasil), administrado sem intercorrências durante $\mathrm{o}$ procedimento. $\mathrm{O}$ paciente foi posicionado em decúbito lateral direito, e introduziu-se uma sonda urinária número 10. Com o auxílio de radiografias, mediante o uso de aparelho de raios $\mathrm{X}$ digital portátil, sistema DR (Cuattro), realizou-se uma uretrocistografia utilizando-se o contraste de Iohexol Hovione (Omnipaque, Farmassa, Xangai, China), diluído com solução isotônica de cloreto de sódio 0,9\% (Fisiológico Linhamax, Eurofarma, Ribeirão Preto, Brasil) para distender a bexiga, mantendo o óstio uretral ocluído manualmente, a fim de que fosse possível avaliar o diâmetro uretral e estimar o tamanho do stent a ser implantado.
Optou-se por utilizar um stent $10 \%$ maior que o diâmetro da uretra íntegra (mensurando a uretra cranial e caudal à área estenosada), de nitinol autoexpansível (Angiolux, Porto Alegre, Brasil), de $40 \mathrm{~mm}$ de comprimento por $8 \mathrm{~mm}$ de diâmetro. Fez-se, então, a sondagem por via retrógrada, acompanhada de um guia hidrofílico e posterior realização de radiografías de controle para confirmar a localização do stent. Quando este foi posicionado no local adequado e compatível com a estenose, foi feita a liberação do stent conforme orientação do fabricante. Na primeira tentativa, o stent foi liberado e migrou para a bexiga devido à falha no posicionamento inicial; na segunda tentativa, foi posicionado na região adequada, o que se confirmou com uma radiografia lateral de controle, e, então, procedeu-se à liberação e expansão do stent, que permaneceu fixo na região de uretra prostática (Fig. 2).

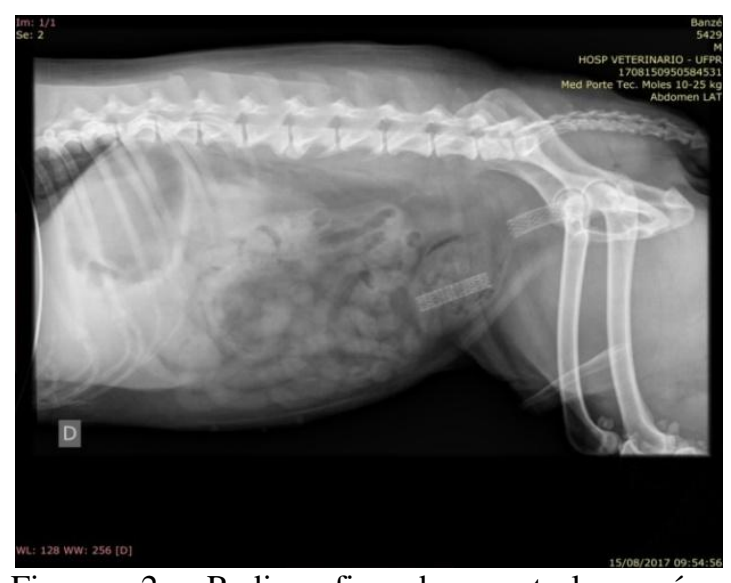

Figura 2. Radiografia de controle após procedimento. Nota-se o primeiro stent liberado antes da posição adequada, o qual migrou para a bexiga, e o stent na uretra prostática mantendo-se fixo no local adequado.

No pós-cirúrgico imediato, o paciente já estava urinando de forma espontânea, apresentando, durante a primeira semana, discreta incontinência urinária, que cessou sem intervenção medicamentosa. Foi realizada nova urinálise com urocultura e antibiograma e houve crescimento profuso de Klebsiella sp. Esse paciente recebeu alta e, na ocasião, estava urinando normalmente. Foi prescrito tratamento para a infecção urinária com amoxicilina com clavulanato de potássio, na dose de $22 \mathrm{mg} \mathrm{kg} \mathrm{BID,} \mathrm{por} 40$ dias, por ser o antibiotico sugerido no antibiograma realizado. Também foi indicado acompanhamento para 
uma nova cistotomia para remoção do stent que migrou para a bexiga.

Não houve o retorno conforme solicitado e também não foi seguida a terapia antibiótica prescrita. Após dois meses do procedimento, esse paciente retornou para nova avaliação. Apresentava micção urinária espontânea, o stent uretral permanecia fixo, como posicionado inicialmente, porém ainda apresentava sinais de infecção no trato urinário inferior. Foi realizada cistotomia para retirada do stent que havia migrado para a bexiga (Fig. 3) e procedeu-se à cultura e ao antibiograma desse material, que demonstrou crescimento profuso de Klebsiella sp. multirresistente. Após esse procedimento, iniciou-se a terapia com ceftriaxona $30 \mathrm{mg} / \mathrm{kg}$ BID. Em contato com o proprietário por telefone,ele relatou que interrompeu a terapia por conta própria e que o paciente estava urinando normalmente.

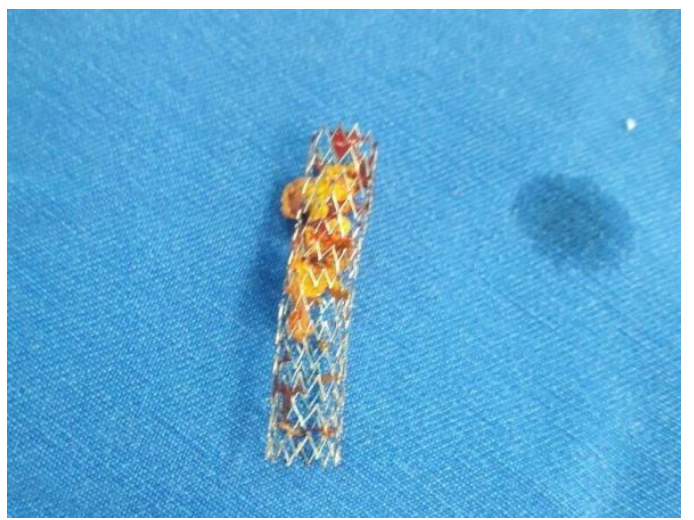

Figura 3. Stent removido da bexiga com presença de debris celulares aderidos ao seu lúmen.

\section{DISCUSSÃO}

Segundo Milroy et al. (1988), a técnica de inserção do stent uretral é simples, embora o tamanho do stent tenha que ser bem avaliado para que não haja complicações como migração. $\mathrm{O}$ procedimento cirúrgico relatado foi rápido e efetivo, mas o acompanhamento da radiografia digital em diversos momentos foi de fundamental importância para que o stent pudesse ser implantado no local correto.

No momento da implantação, Radhakrshnan (2017) confirma que a fluoroscopia é o melhor método de imagem para se utilizar, porém o procedimento pode ser realizado com sucesso pela radiografia digital, assim como foi feito no caso descrito. A radiografia trouxe um excelente auxílio e possibilitou a aferição do diâmetro uretral, bem como a aplicação do stent sem a necessidade da fluoroscopia. A sua principal limitação é a necessidade de múltiplas radiografias, desde a uretrocistografia de contraste, o posicionamento do fio guia até a implantação do stent. Essas limitações podem aumentar o risco de complicações e, por isso, devem ser realizadas com imagem adequada e progressão cuidadosa para se ter um bom resultado.

Para assegurar uma posição correta do stent, ele foi implantado sendo $10 \%$ mais largo do que o diâmetro da uretra adjacente cranial e caudal à regiao estenosada. O comprimento do stent se estende $1 \mathrm{~cm}$ cranialmente e $1 \mathrm{~cm}$ caudalmente à área estenosada, como relatado por Weisse et al. (2006), sendo o ideal para evitar migrações. Esses pesquisadores realizaram um estudo com 12 cães, e $90 \%$ deles foram capazes de urinar de modo voluntário, imediatamente após a implantação do stent uretral, assim como no paciente relatado, que urinou no pós-cirúrgico imediato, função essa que não conseguia realizar sem o auxílio da sonda há 30 dias. O sinal de incontinência urinária na primeira semana pode estar relacionado com a localização da estenose na uretra prostática, pois essa região de terço proximal da uretra é composta pela membrana prostática, que, segundo Weisse et al, (2006), é considerada a zona de continência da uretra. Essa incontinência pode ser temporária ou permanente, e, no caso relatado, o paciente voltou a urinar normalmente em uma semana de pós-operatório.

Latal et al. (1994) mostram que o stent de nitinol tem uma excelente biocompatibilidade. Ko et al. (2002) relatam riscos de complicações, como fratura do stent, e migração sendo menos frequente, porque ele exerce uma força radial sobre a parede da uretra e o material do stent é formado de um único fio de nitinol. No presente relato, houve a migração do stent devido à falha no posicionamento inicial, porém o segundo stent, que foi devidamente posicionado, permaneceu fixo, sem sinais de migração, até o presente momento (quatro meses de pósoperatório). Choi et al. (2007) relatam que a migração é o maior obstáculo para se alcançar a duração adequada do stent, sendo a implantação 
a parte mais crítica para que sejam obtidos bons resultados.

Hill et al. (2014) descreveram um acompanhamento em longo prazo, por dois anos, de cães com estenose uretral e comprovaram o sucesso do tratamento, ao verificarem ser este seguro, eficaz e duradouro. Nesses pacientes, $82 \%$ dos cães apresentaram cistite bacteriana no momento do diagnóstico, o que enfatiza a necessidade de urinálise e urocultura para todos os cães que irão ser submetidos a tal procedimento. Esses cães passaram por tratamento com antibióticos baseados no antibiograma por um período de quatro a seis semanas. Latal et al. (1994) relatam boa biocompatibilidade e ausência de infecções em um estudo realizado a curto prazo.

No presente relato, o paciente apresentava infecção do trato urinário desde o princípio do atendimento.Foi iniciada a terapia indicada pelos exames de antibiograma, e, após a implantação do stent, foi prescrito um tratamento longo, de 40 dias, conforme preconiza a literatura. Porém, a terapia indicada não foi seguida, o que fez com que piorasse o quadro de infecção urinária. Ao voltar para atendimento, o paciente apresentava uma cistite multirresistente. Essa falha na terapia e o fato de o stent ter sido implantado quando o paciente ainda apresentava infecção urinária são fatores que contribuíram para a perpetuação da infecção, sendo o indicado realizar o tratamento para infecção do trato urinário antes de se colocar o stent.

\section{CONCLUSÃO}

O uso do stent endovascular no caso citado trouxe benefícios ao paciente, visto que ele era dependente da sondagem para conseguir urinar. Essa técnica se mostrou viável com o uso da radiografia digital e, quando implantada de forma adequada, no diâmetro e na região anatômica preconizada, reduz a chance de complicações. Porém, os cuidados e a monitorização devem ser constantes para se evitarem riscos de complicações.

\section{REFERÊNCIAS}

BRACE, M.A.; WEISSE, C.; BERENT, A. Preliminary experience with stenting for management of non-urolith urethral obstruction in eight cats. Vet. Sur., v.43, p.199-208, 2013.

CHOI, E.K.; SONG, H.Y.; SHIN, J.H. et al. Management of recurrent urethral strictures with covered retrievable expandable nitinol stents: long-term results. Am. J. Roentgenol., v.189, p.1517-1522, 2007.

CHOI, R.; LEE, S.; HYUN, C. Urethral stenting in a cat with refractory obstructive feline lower urinary tract disease. J. Vet. Med. Sci., v.71, p.1255-1259, 2009.

DYER, R.B.; CHEN, M.Y.; ZAGORIAET, R.J. et al. Complications of ureteral stent. Radiographic, v.22, p.1005-1022, 2002.

HILL, T.L.; BERENT, A.C.; WEISSE, C.W. Evaluation of urethral stent for benign urethral obstructions in dogs. J. Vet. Intern. Med., v.28, p.1384-1390, 2014.

KO, G.Y.; KIM, G.C.; SEO, T.S. et al. Covered, retrievable, expandable urethral nitinol stent: Feasibility study in dog. Radiology, v.223, p.8389, 2002.

LATAL, D. MRAZ, J.; ZERHAU, P. et al. Nitinol urethral stents: long-term results in dogs. Urol., Res., v.22, p.295-300, 1994.

LIATSIKOS, E.N.; KARRNABATIDIS, D.; KAGADIS, G.C. et al. Metal stents in the urinary tract. Eur. Assoc. Urol. Eur. Board. Urol., v.5, p.77-88, 2007.

MILROY, E.J.G.; CHAPPLE, C.R.; COOPER, J.E. et al. A new treatment for urethral strictures. Lancet, v.331, p.1424-1427, 1988.

RADHAKRSHNAN, A. Urethral stenting for obstructive uropathy utilizing digital radiography for guidance: feasibility and clinical outcome in 26 dogs. J. Vet. Intern. Med., v.31, p.427-433, 2017.

WEISSE, C.; BERENT, A.; TODD, K. et al. Evaluation of palliative stenting for management of malignant urethral obstructions in dogs. J. Am. Vet. Med. Assoc., v.229, p.226-234, 2006. 